\title{
Metabolic characteristics of taste differences under the soil and hydroponic cultures of sweet potato leaves by using non-targeted metabolomics
}

\author{
Zhaomiao Lin, Guoliang Li, Hong Zhang, Rongchang Ji, Yongqing Xu, Guochun Xu, Huawei Li, Zhonghua Liu, \\ Wenbin Luo, Yongxiang Qiu, Sixin Qiu and Hao Tang* \\ Institute of Crop Sciences, Fujian Academy of Agricultural Sciences, Scientific Observing and Experimental Station \\ of Tuber and Root Crops in South China, Ministry of Agriculture and Rural Affairs. Fuzhou, Fujian 350013, China. \\ *Author for correspondence (tanghao9403@163.com)
}

\begin{abstract}
Sweet potato leaves are consumed as green leafy vegetables in most of the world due to their nutritional and functional values, and the taste characteristics determine their commodity value and consumer acceptance. However, the metabolic composition and formation mechanism of taste quality in its leaves are not clear. In this study, we found that sweet potato leaves under different growing patterns, soil culture and hydroponic culture, which result in different taste quality. In particular, the taste quality in leafy sweet potato was effectively improved under hydroponic culture. Meanwhile, we further profiled metabolites in leaves of sweet potatoes under different growing patterns by using GC-QToF-MS. A total of 200 metabolites were identified, covering most of the metabolic pathways in plants. A comparison of the good taste and poor taste of sweet potato leaves resulted in 71 metabolites related to taste quality formation. In addition, the leaves with poor taste had lower levels of metabolites regarding amino acids metabolism, whereas was accompanied by high levels of metabolites in carbohydrates and secondary metabolism. This study provides new insights into the improvement of taste quality in leafy sweet potato.
\end{abstract}

KEY WORDS: Sweet potato; leaves; taste quality; metabolomic; soil and hydroponic cultures

\section{INTRODUCTION}

Sweet potato (Ipomoea batatas L.) is an important food crop in the world, with its roots be used for food or raw materials in industrial production. Meanwhile, sweet potato leaves, the young parts of the shoot in sweet potatoes, have been used as leafy vegetables in most tropical and subtropical areas (Islam, 2006). This vegetable-use of sweet potato was called leafy sweet potato. Compared with ordinary leafy vegetables, leafy sweet potato have many advantages. Such as, their yield is high due to can be harvested several times a year (Sun et al., 2016); and their nutritional and functional values were much higher than its root or other leafy vegetables (Dinu et al., 2018; Li et al., 2019; Sun et al., 2014); as well as they have a strong resistance to drought, waterlogging, and barren, etc (Sui et al., 2019).

Taste quality is one of the important quality traits of vegetables. In leafy sweet potato, the taste characteristics determine its commodity value and consumer acceptance (Lee et al., 2007; Ona and Chujoy, 1991). It has been reported that hydroponic culture increased vitamin C, flavone, nitrate contents, and yield of sweet potato leaves compared with soil culture (Chen et al., 2013). In the previous study, compared to soil grown strawberries, the hydroponically grown strawberries were wined preferred by $70 \%$ of participants in the sensory analysis (Treftz et al., 2015). We also found that hydroponic culture also can effectively improve the taste quality in leafy sweet potato, especially the cultivars with poor taste under soil culture in the field. However, the metabolic composition and formation mechanism of taste quality in its leaves are not clear.

Metabolomics is the modern instrumental analysis method with high-throughput, high-resolution, 
and high-sensitivity, to comprehensively study the various features of endogenous metabolites in living organisms (Saito and Matsuda, 2010). For taste quality analysis, metabolomic approaches have yielded new insights into the taste change in the breeding history of tomato (Zhu et al., 2018), the sensory quality assessment of garlic (Liu et al., 2019), exploring the relationships between these substances and tastes of white tea (Yang et al., 2018), and Identification of key taste components in loquat (2020). Therefore, in this study, we used the untargeted metabolomic analysis of sweet potato leaves under different growing patterns, soil culture and hydroponic culture, for exploring the metabolic foundation of different taste quality in sweet potato leaves.

\section{RESULTS}

\section{Differences of taste quality in sweet potato leaves under different growing patterns}

Leafy sweet potato is a special type of sweet potato for vegetables, and the taste quality is the one of major concerned trait for the market and consumer. Leafy sweet potato cultivation is mainly based on traditional land cultivation methods. However, use of hydroponic culture to produce leafy sweet potato has many advantages for shoot growth and its yield increase, because it goes against root formation in nutrient solution (Chen et al., 2013). Hydroponic culture is also beneficial for the pollution-free, standardized, and mechanized production of leafy sweet potato. In our preexperiment, we compared the taste characteristics of the leaves of 8 different sweet potato varieties under hydroponic culture $(\mathrm{H})$ and soil culture $(\mathrm{S})$ conditions, and found that hydroponic culture can effectively improve the taste quality of sweet potato leaves, especially the cultivars with poor taste under soil culture (Table S1). Thus, hydroponics can be used as a reliable cultivation mode to improve the taste quality of leafy sweet potatoes. In addition, the taste quality of Baisheng under soil culture was poor, while the taste quality of Baisheng under hydroponic culture (Baisheng-S) was significantly improved; and taste quality of Fucaishu18 was a small change under the different growing patterns (Fig. 1). Among them, the latter three samples (Baisheng-H, Fucaishu18-S, and Fucaishu18-H) have similar taste values. Therefore, Fucaishu1 8 and Baisheng were chosen and used for the analysis of the metabolites related to taste traits by using non-targeted metabolomics.

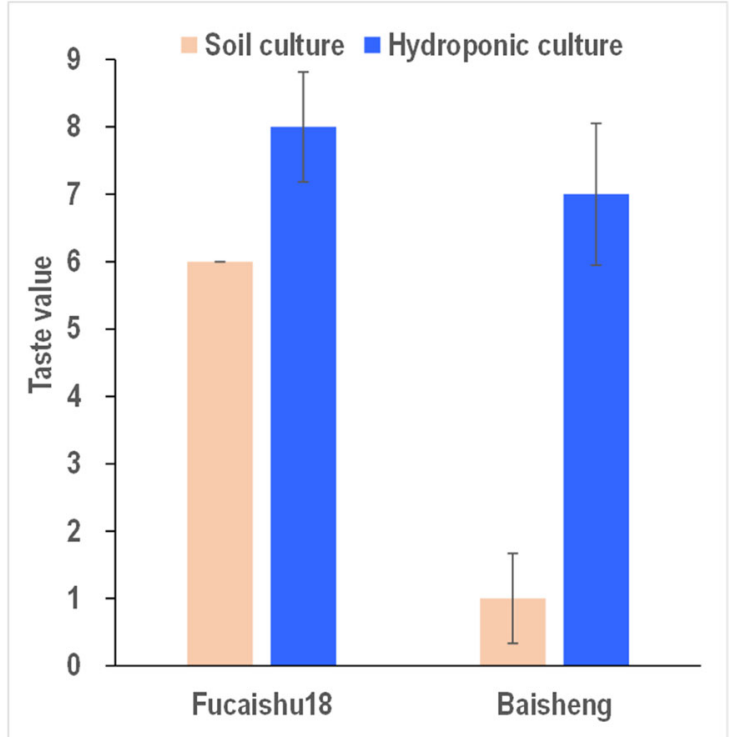

Fig. 1. Taste values of leaves in Baisheng and Fucaishu18 under different growing patterns.

\section{Metabolite profiling of leaves in sweet potato}


By subjecting the leaves of sweet potato growth in soil and hydroponic cultures produced in the plastic house to GC-TOF-MS analysis, 518 peaks were detected and 200 metabolites could be identified by searching against LECO-Fiehn Rtx 5 database (Fig. 2A). In this experiment, 5 quality control samples (QC) were used for ensuring data re-liability, and the result showed high repeatability (Fig. S1, S2) and concordance (Fig. S3). The 200 metabolites could be assigned into 7 super pathways (Fig. 2B) and sub-sequent 30 sub-pathways (Table S2) according to Kyoto Encyclopedia of Genes and Genomes (KEGG). These metabolites covered mainly the central metabolism pathways and partial secondary metabolism pathways, including 45 amino acids, 94 carbohydrates, 28 lipids, 5 CPGECs (cofactors, prosthetic groups, electron carriers), 5 nucleotides, 22 secondary metabolites, and 1 phytohormone.
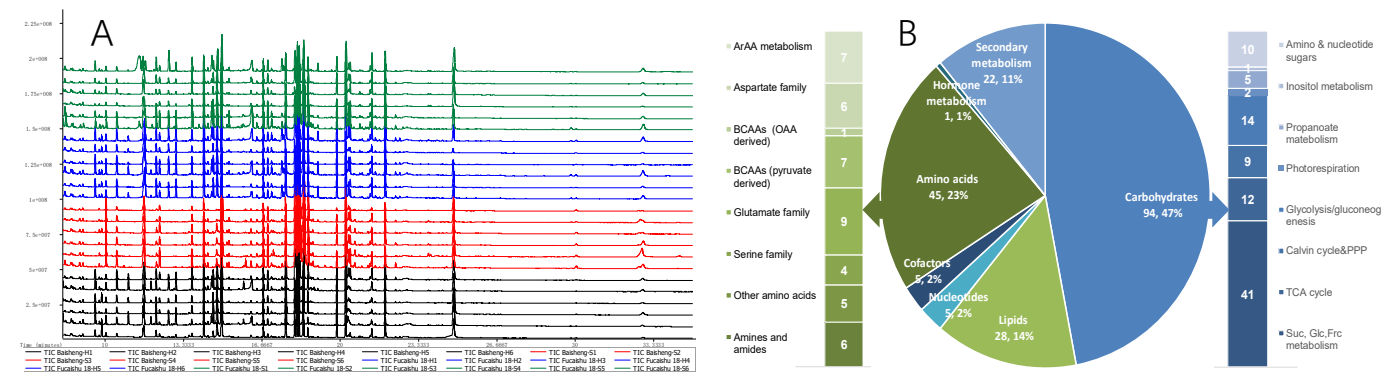

Fig. 2. GC-TOF-MS analysis and classification of the identified metabolites in sweet potato

leaves. A, Total Ions Chromatograph (TIC) of 4 samples with 6 replicates in sweet potato leaves via GC-TOF-MS. Green and blue graphs indicate the samples of Fucaishu18-S (soil culture) and Fucaishu18- H (hydroponic culture), respectively. red and black graphs indicate the samples of Baisheng-S (soil culture) and Baisheng-H (hydroponic culture), respectively. B, Distribution of identified 200 metabolites in pie chart, sub-classifications of amino acids and carbohydrates are shown with histograms.

A non-supervised principal component analysis (PCA) was performed with all 200 metabolites. As shown in Fig. 3, there was a clear separation among the 4 samples, indicating that the metabolome of Baisheng growth under soil culture (Baisheng-S, that was poor taste) was distinct from that of the other 3 samples including Baisheng-H, Fucaishu18-S, and Fucaishu18-H. Meanwhile, a heat map display of all data showed a difference cluster mode in relative abundances of Baisheng-S among the 4 samples (Fig. 4). This is the expected pattern as the result both of PCA and cluster analysis is consistent with the change in taste value between soil and hydroponic cultures of two cultivars (Fig. 1). Note that in the complexity of metabolite changes among the 4 samples, we chose 71 metabolites that the abundance was different at Baisheng-S com-pare with the other 3 samples at the same abundance, for future pathway analysis.

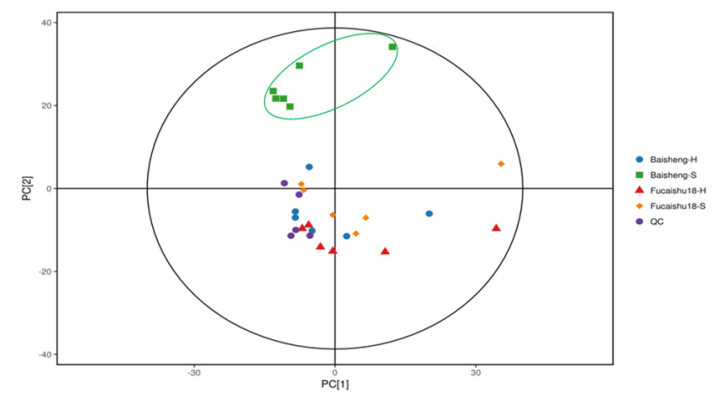

Fig. 3. Score scatter plot of PCA model for metabolites (peaks) in leaves of Fucaishu18 and 
Baisheng under different growing patterns. H, hydroponic culture; S, soil culture; QC, quality control.

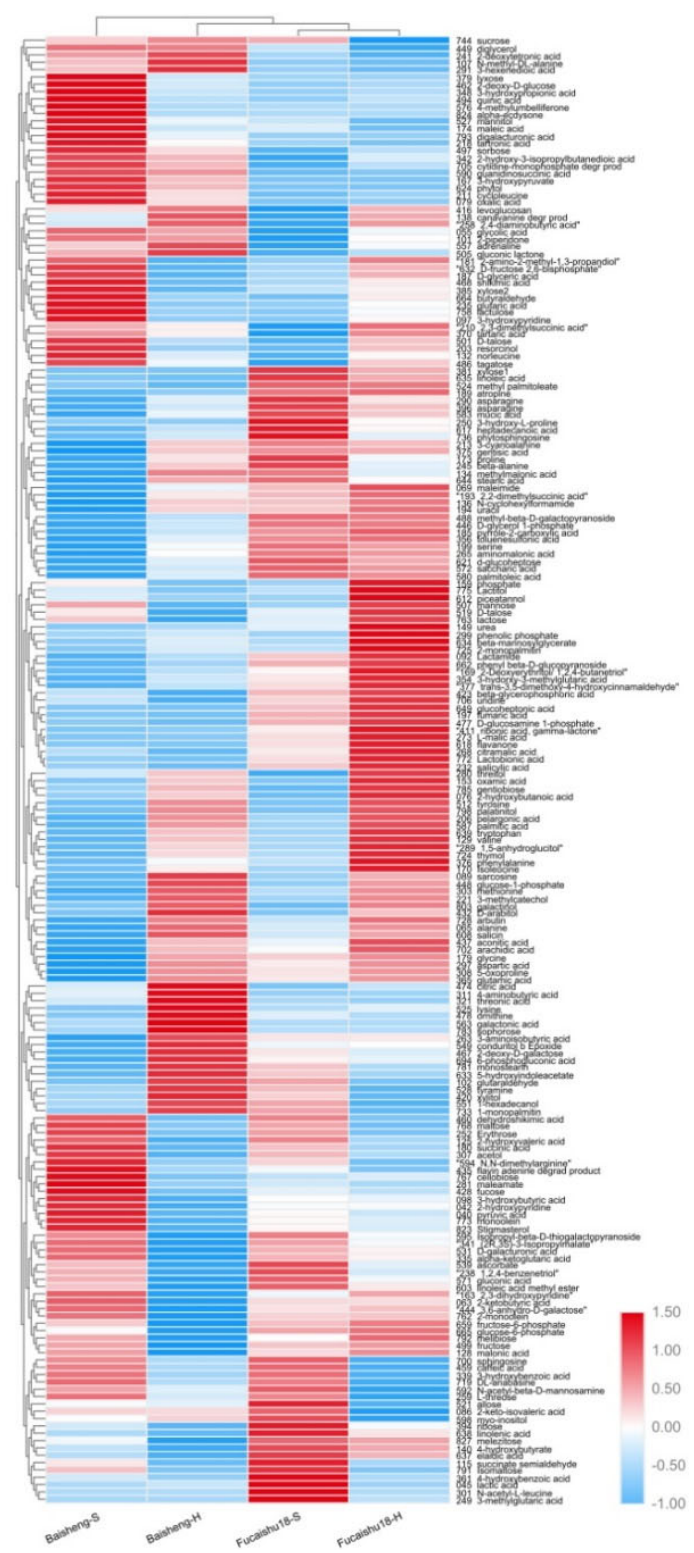

Fig. 4. Heat map presentation of the variation of the 200 metabolites in leaves of Baisheng and Fucaishu18 under different growing patterns. H, hydroponic culture; S, soil culture. Each metabolite abundances in 4 samples were normalized with Z-score analysis.

\section{Metabolites and their pathway related to taste quality in sweet potato leaves}

Reduced amino acids abundance in Baisheng-S with poor taste

Amino acid metabolism is a major part of metabolic networks in biological systems; meanwhile, the amino acids and their derivatized into numerous secondary metabolites, which are the bioactive or flavor substance in food (Kays and Wang, 2000; Lindsay, 2017). In this study, we detected 18 metabolites involve in amino acid metabolism. Except for N, N-dimethylarginine (ADMA), 3cyanoalanine, N-amidino-L-aspartate, cycloleucine, the Baisheng-S contained a lower abundance of other 14 amino acids and their derivatives. All of four umami amino acids including Asp, Glu, Gly, and Ala were lower abundance in Baisheng-S, especially (Fig. 5, Table S2). 


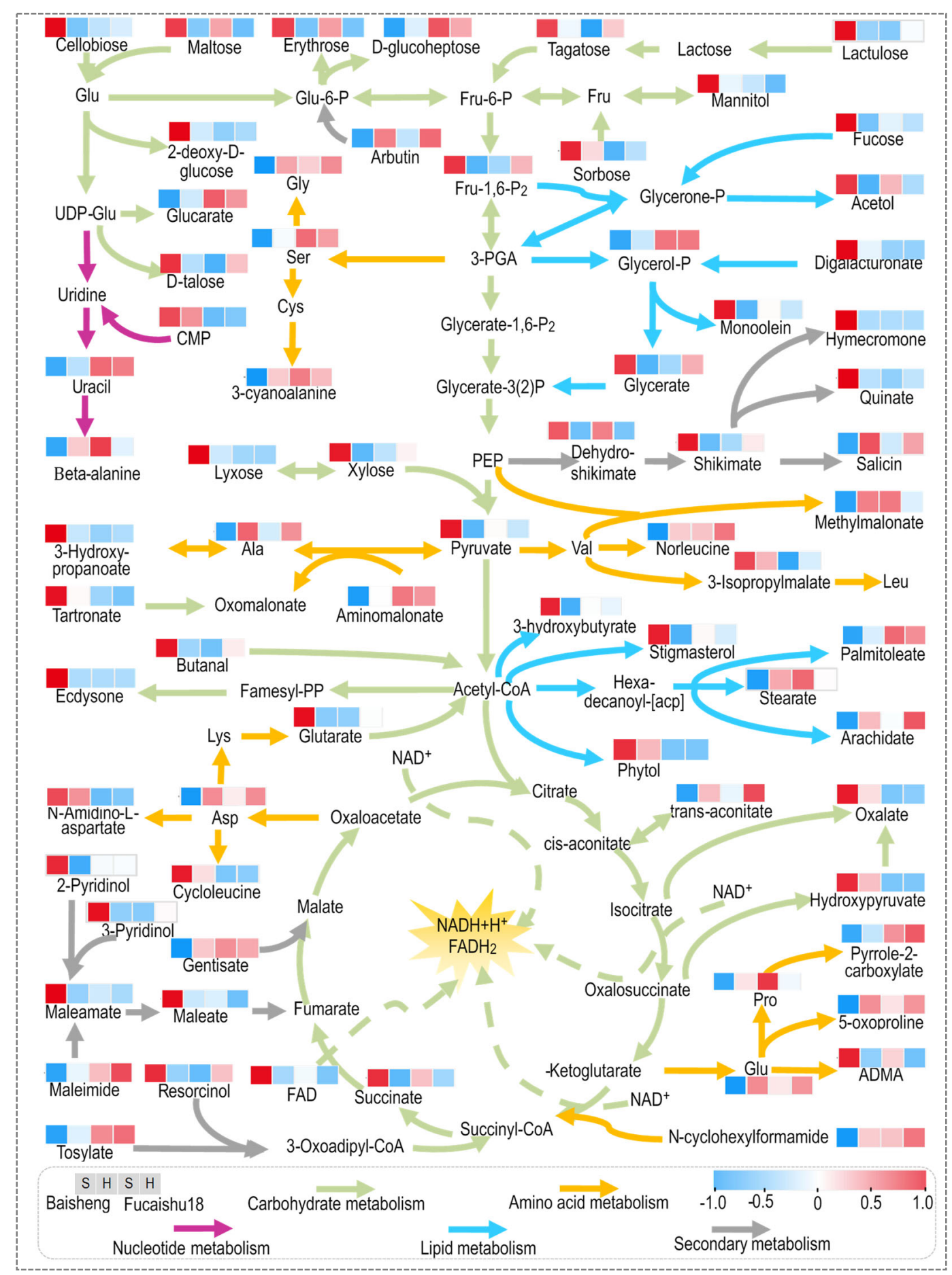

Fig. 5. Metabolite abundances in leaves of Baisheng and Fucaishu18 under different growing patterns. The four squares under the names of metabolites indicate abundance change of BaishengSoil culture(S), Baisheng-Hydroponic culture(H), Fuchaishu18-Soil culture(S), Fuchaishu18Hydroponic culture $(\mathrm{H})$, respectively. Metabolite abundances of 4 samples were normalized with Zscore analysis. A red square indicates high abundance, whereas a blue square indicates low abundance.

Enhanced lipids synthesis, but not for ester synthesis

Plants lipids are important components in cell membrane, signal molecules, and stored energy. In 
this study, 10 metabolites related to lipids metabolism, that including derived from acetyl-CoA and 3-PGA. Seven of them were increased abundance in Baisheng-S, but three esters including stearate, arachidate, and palmitoleate were de-creased (Fig. 5, Table S2).

Increased secondary metabolism and accumulate

Plant secondary metabolites, also referred to as natural products or specialized metabolites, constitute an enormously rich reservoir of chemical biodiversity. They are provided most of the favor substances and bioactive metabolites for human health in vegetables. Thirteen secondary metabolites were detected change in Baisheng-S, among them, 9 metabolites were increased. Notably, the most important pathway for secondary metabolism, the shikimate metabolic pathway was enhanced (Fig. 5, Table S2). This suggests that secondary metabolites were accumulated and associated with worse taste.

\section{DISCUSSION}

Metabolomics is a powerful tool for studying the quality traits of crops (Liu et al., 2019; Zhu et al., 2018; Zou et al., 2020). In previous studies, metabolomics has been used successfully for quality study in sweet potato, including anthocyanin accumulation in tuberous roots, metabolic mechanism of flesh color difference, metabolic change during postharvest storage, and metabolic diversity in leaves and roots (Wang et al., 2018; Drapal et al., 2019; He et al., 2020; Ren et al., 2021). However, the taste quality of sweet potato leaves has not been investigated so far. In this study, we used GCQToF-MS-based untargeted metabolomic to analyze taste differences under the soil and hydroponic cultures of sweet potato leaves. A total of 200 metabolites were identified, and 71 metabolites of which were related to flavor formation in sweet potato leaves. Thus, this study provides a metabolic reference of taste quality in leafy sweet potato.

Metabolites are essential components in plants, and they can be classified as carbohydrates, organic and amino acids, vitamins, hormones, flavonoids, phenolics, and glucosinolates (Hounsome et al., 2008; Mabuchi et al., 2019). Some of these metabolites can determine the taste of leafy vegetables. Free amino acids can provide a variety of taste characteristics in leafy vegetables, such as glycine and alanine are sweetness, valine, tryptophan, and leucine are bitterness, and aspartic acid and glutamate have a sour taste (Ardö, 2006; Hounsome et al., 2008; Mabuchi et al., 2019; Toko, 2000). Another major group of sub-stances that determine taste is secondary metabolites, including flavonoids, phenolics, and alkaloids. They are the main source of bitterness and astringency (Mabuchi et al., 2019; Peterson and Dwyer, 1998; Streit et al., 2007). Our results in agreement with these above studies. Thus, we speculate that, the reason for the poor taste of sweet potato leaves under soil culture is the lower levels of metabolites regarding amino acids metabolism, whereas was accompanied by high levels of metabolites in carbohydrates and secondary metabolism. Therefore, further research should focus on the dynamic metabolic characteristics of amino acids and secondary metabolites, to explore the formation mechanism of the taste quality in leafy sweet potato, and the agronomic measures to improve the taste quality.

\section{MATERIALS AND METHODS}

\section{Plant Growth and Sample Collection}

The experiments were performed at the Pudang agricultural experiment farm in Fujian Academy of Agricultural Sciences $\left(26^{\circ} 07^{\prime} 59^{\prime \prime}\right.$ N, 119 $20^{\prime} 06^{\prime \prime}$ E), Fuzhou City, China. To explore the metabolic mechanisms of taste quality of sweet potato leaves, two sweet potato cultivars including Fucaishu18 
and Baisheng was used as experimental material with two types of growing patterns including hydroponic culture and soil culture under the plastic house.

Hydroponic culture $(\mathrm{H})$. Eight plants in a container $(40 \mathrm{~cm} \times 28 \mathrm{~cm} \times 15 \mathrm{~cm})$ of two cul-tivars were growing in half-strength Hoagland solution couple with suitable micro-nutrients (Hoagland and Arnon; 1950), and pumping air for 5 minutes every two hours in the nutrient solution.

Soil culture (S). A pot experiment was conducted using plastic pots, $15 \mathrm{~cm}$ in height and $12 \mathrm{~cm}$ in diameter. Each pot was filled with $1.5 \mathrm{~kg}$ clay soil, containing $0.83 \mathrm{~g} \mathrm{~kg}^{-1}$ total $\mathrm{N}, 10.72 \mathrm{mg} \mathrm{kg}^{-1}$ available $\mathrm{P}$, and $69.15 \mathrm{mg} \mathrm{kg}-1$ exchangeable $\mathrm{K}$. For one pot, five plants of sweet potatoes were hand transplanted. The basal fertilization before trans-planting used $0.20 \mathrm{~g} \mathrm{~N}, 0.24 \mathrm{~g} \mathrm{P}_{2} \mathrm{O}_{5}$, and 0.9 $\mathrm{g} \mathrm{K}_{2} \mathrm{O}$ per pot.

At the 30 days after transplanted, the last leaves with fully expanded form five plants were collected from Fucaishu18 and Baisheng under different growing patterns. The four samples were named Fucaishu18-S (soil culture), Fucaishu18- H (hydroponic culture), Baisheng-S (soil culture), and Baisheng-H (hydroponic culture), respectively.

Leaves were frozen by liquid nitrogen and then stored at $-80^{\circ} \mathrm{C}$ until analysis. Each sample was measured with six biological replicates.

\section{Sensory Analysis}

According to the practice method from the experienced breeder of leafy sweet potato, a sensory panel of 10 trained panelists was selected in the evaluation of sensory proper-ties, with the methodology modified by Ona et al (1991) and Ishiguro et al (2004). Samples of sweet potato leaves were cooked for 2 minutes by boiling water, then evaluated imme-diately with four aspects including flavor, sweetness, bitterness, and crispiness. The leaves of Fuchaishu18 growth in soil were used as the standard with defining its evalu-ation score as 6, and other samples have a score range of 0-9 from the worst to the best by sensory of each panel.

\section{Metabolomic Analysis}

\section{Metabolites Extraction and GC-MS Analysis}

Metabolite profiling was performed by Shanghai Biotree Biotech Co., Ltd. in China, according to the method of Wu et al. (2019). Briefly, take $60 \mathrm{mg}$ sample into the $2 \mathrm{~mL}$ EP tubes, extracted with $0.48 \mathrm{~mL}$ extraction liquid (VMethanol: $\mathrm{VH} 2 \mathrm{O}=3: 1)$, add $20 \mu \mathrm{L}$ of adonitol $(0.5 \mathrm{mg} / \mathrm{mL}$ stock in $\mathrm{dH} 2 \mathrm{O}$ ) as internal standard, vortex mixing for 30s; Homogenized in ball mill for $4 \mathrm{~min}$ at $45 \mathrm{~Hz}$, then ultrasound treated for $5 \mathrm{~min}$ (incubated in ice water); Centrifuge for 15 minat $12000 \mathrm{rpm}, 4^{\circ} \mathrm{C}$; Transfer the supernatant $(0.35 \mathrm{~mL})$ into a fresh $2 \mathrm{~mL} \mathrm{GC} / \mathrm{MS}$ glass vial, take $40 \mu \mathrm{L}$ from each sample and pooling as QC sample. Dry completely in a vacuum concentrator without heating; Add $80 \mu \mathrm{L}$ Methoxyamination hydrochloride $\left(20 \mathrm{mg} / \mathrm{mL}\right.$ in pyridine) incubated for $30 \mathrm{~min}$ at $80^{\circ} \mathrm{C}$; Add $100 \mu \mathrm{L}$ of the BSTFA regent $(1 \%$ TMCS, $v / v)$ to the sample aliquots, incubated for $1.5 \mathrm{~h}$ at $70^{\circ} \mathrm{C}$; Add $10 \mu \mathrm{L}$ FAMEs (Standard mixture of fatty acid methyl esters, C8-C16:1mg/mL; C18-C24:0.5mg/mL in chloroform) to the QC sample when cooling to the room temper-ature; All samples were analyzed by gas chromatography system (Agilent 7890) coupled with a Pegasus 4D time-of-flight mass spectrometer (GC-TOF-MS).

\section{Data Analysis and Annotation}

Chroma TOF 4.3X software of LECO Corporation and LECO-Fiehn Rtx5 database were used for raw peaks exacting, the data baselines filtering and calibration of the baseline, peak alignment, deconvolution analysis, peak identification, and integration of the peak area (Kind et al., 2009) . Both mass spectrum match and retention index match were considered in metabolites identification. 
Remove peaks detected in $<50 \%$ of QC samples or RSD $>30 \%$ in QC samples (Dunn et al., 2011) . Besides, internal standard normalization method was employed in this data analysis. The resulted three-dimensional data involving the peak number, sample name, and normalized peak area were fed to SIMCA software package (V14.1, MKS Data Analytics Solutions, Umea, Sweden) for principal component analysis (PCA) and orthogonal projections to latent structures-discriminate analysis (OPLS-DA). Public databases including KEGG (http://www.genome.jp/kegg/) and MetaboAnalyst (http://www.metaboanalyst.ca/) was utilized to search for the pathways of metabolites.

\section{Competing interests}

The authors declare no competing or financial interests.

\section{Author contributions}

Methodology, Z.L. (Zhaomiao Lin) and G.L.; software, G.L.; investigation, G.L., G.X. and Y.X.; resources, Z.L. (Zhonghua Liu), R.J., W.L. and G.X.; writing-review and editing, Z.L. (Zhaomiao Lin), G.L., H.Z., G.X., and S.Q.; visualization, Z.L. (Zhaomiao Lin); supervision, Y.Q., S.Q., and H.T.; funding acquisition, S.Q. and H.T. All authors have read and agreed to the published version of the manuscript.

\section{Funding}

This work was supported by the Fujian Provincial Public Research Institute of Fundamental Research (2017R1026-5), the Special Fund for the Industrial Technology System Construction of Modern Agriculture of China (CARS-10-B14), the Fujian Provincial Department of Science \& Technology (2017NZ0002-2), and the Fujian Academy of Agricultural Sciences Research Project (AGP2018-12).

\section{Supplementary information}

Fig. S1: Total Ions Chromatograph (TIC) of 5 Quality Control Samples, 5 Internal Standards in QC samples, and Blank sample; Fig. S2: Principal Component Analysis plot of the peaks in 5 QC samples and 24 test samples; Fig. S3: High concordance among the peaks in five QC samples (r2 $\geq 0.941$ ). Fig. S4: Score scatter plot of OPLS-DA model for metabolites (peaks) in leaves of Fucaishu18 and Baisheng under different growing patterns; Fig. S5: Pathway analysis for different metabolites in leaves of Baisheng and Fucaishu18 under different growing patterns. Table S1: Taste values of leaves in eight cultivars under different growing patterns; Table S2: Heat map of metabolite abundance in leaves of sweet potato. 
bioRxiv preprint doi: https://doi.org/10.1101/2021.02.24.432602; this version posted February $25,2021$. The copyright holder for this

preprint (which was not certified by peer review) is the author/funder, who has granted bioRxiv a license to display the preprint in perpetuity. It is made available under aCC-BY-NC-ND 4.0 International license.

\section{References}

Ardö, Y. (2006). Flavour formation by amino acid catabolism. Biotechnol Adv. 24, 238-242.

Chen, X.Y., Zhang, Z.J., Zheng, J.W. and Lin, Y.L. (2013). Effects of hydroponics on nutrient components and nitrate contents in tips of leaf vegetable sweet potato. Sci. Agric. Sin. 46, 3736-3742. (in Chinese with English abstract).

Dinu, M., Soare, R., Băbeanu, C. and Hoza, G. (2018). Analysis of nutritional composition and antioxidant activity of sweet potato (Ipomoea batatas L.) leaf and petiole. J. Appl. Bot. Food Qual. 91, 120-125.

Drapal, M., Rossel, G., Heider, B. and Fraser, P.D. (2019). Metabolic diversity in sweet potato (Ipomoea batatas, Lam.) leaves and storage roots. Hortic. Res. 6, 2.

Dunn, W.B., Broadhurst, D., Begley, P., Zelena, E., Francis-McIntyre, S., Anderson, N., Brown, M., Knowles, J.D., Halsall, A., Haselden, J.N., Nicholls, A.W., Wilson, I.D., Kell, D.B., Goodacre, R. and Human Serum Metabolome (HUSERMET) Consortium. (2011). Procedures for large-scale metabolic profiling of serum and plasma using gas chromatography and liquid chromatography coupled to mass spectrometry. Nat. Protoc. 6, 10601083.

He, L., Liu, X., Liu, S., Zhang, J., Zhang, Y., Sun, Y., Tang, R., Wang, W., Cui, H., Li, R., Zhu, H. and Jia, X. (2020). Transcriptomic and targeted metabolomic analysis identifies genes and metabolites involved in anthocyanin accumulation in tuberous roots of sweetpotato (Ipomoea batatas L.). Plant Physiol. Biochem. 156, $323-332$

Hounsome, N., Hounsome, B., Tomos, D. and Edwards-Jones, G. Plant metabolites and nutritional quality of vegetables. J Food Sci. 2008, 73, R48-65.

Hoagland, D.R. and Arnon, D.I. (1950). The water-culture method for growing plants without soil. Circular \& California agricultural experiment station. 347, 32 .

Kays, S.J. and Wang, Y. (2000). Thermally induced flavor compounds. HortScience, 35, 1002-1012.

Kind, T., Wohlgemuth, G., Lee, D.Y., Lu, Y., Palazoglu, M., Shahbaz, S. and Fiehn, O. (2009). FiehnLib: mass spectral and retention index libraries for metabolomics based on quadrupole and time-of-flight gas chromatography/mass spectrometry. Anal. Chem. 81, 10038-10048.

Lee, J.S., Ahn, Y.S., Chung, M.N., Kim, H.S., Jeong, B.C. and Bang, J.K. (2007). Varieties for sweetpotato tips production as a vegetable. Korean J. Breeding Sci. 39, 224-231.

Li, G., Lin, Z., Zhang, H., Liu, Z., Xu, Y., Xu, G., Li, H., Ji, R., Luo, W., Qiu, Y., Qiu, S. and Tang, H. (2019). Anthocyanin Accumulation in the Leaves of the Purple Sweet Potato (Ipomoea batatas L.) Cultivars. Molecules. 24, 3743.

Lindsay, R.C. (2017). Flavors. In Fennemas Food Chemistry, 5th. (ed., Damodaran, S; Parkin, K.L.), pp. 753-802. Boca Raton: CRC Press.

Liu, J., Liu, L.X., Guo, W., Fu, M.L., Yang, M.L., Huang, S.X., Zhang, F. and Liu, Y.S. (2019). A new methodology for sensory quality assessment of garlic based on metabolomics and an artificial neural network. Rsc Advances. 9, 17754-17765.

Ishiguro, K., Toyama, J., Shahidul Islam, M.D., Yoshimoto, M., Kumagai, T., Kai, Y., Nakazawa, Y. and Yamakawa, O. (2004). Suioh, a new sweetpotato cultivar for utilization in vegetable greens. Acta Hortic. 637, 339-345.

Islam, S. (2006). Sweetpotato (Ipomoea batatas L.) leaf: its potential effect on human health and nutrition. J. Food Sci. 71, R13-R121.

Mabuchi, R., Tanaka, M., Nakanishi, C., Takatani, N. and Tanimoto, S. (2019). Analysis of Primary Metabolites in Cabbage (Brassica oleracea var. capitata) Varieties Correlated with Antioxidant Activity and Taste Attributes by Metabolic Profiling. Molecules. 24, 4282.

Ona, C.L. and Chujoy, E. (1991). Screening of sweet potato for leaf tips as vegetable. Working Papers of International Potato Center, pp. 194-204.

Peterson, J. and Dwyer, J. Flavonoids: dietary occurrence and biochemical activity. Nutr. Res. 1998, 18,1995-2018.

Ren, L., Zhang, T., Wu, H., Ge, Y., Zhao, X., Shen, X., Zhou, W., Wang, T., Zhang, Y., Ma, D. and Wang, A. (2021). Exploring the metabolic changes in sweet potato during postharvest storage using a widely targeted metabolomics approach. J. Food Process. Pres. 45, e15118. 
bioRxiv preprint doi: https://doi.org/10.1101/2021.02.24.432602; this version posted February $25,2021$. The copyright holder for this

preprint (which was not certified by peer review) is the author/funder, who has granted bioRxiv a license to display the preprint in perpetuity. It is made available under aCC-BY-NC-ND 4.0 International license.

Saito, K. and Matsuda, F. (2010). Metabolomics for functional genomics, systems biology, and biotechnology. Annu. Rev. Plant Biol. 61, 463-489.

Streit, N.M., Hecktheuer, L.H.R., do Canto, M.W., Mallmann, C.A., Streck, L., Parodi, T.V. and Canterle, L.P. (2007). Relation among taste-related compounds (phenolics and caffeine) and sensory profile of erva-mate (Ilex paraguariensis). Food Chem. 102,560-564.

Sui, W., Mu, T., Sun, H. and Yang, H. (2019). Effects of different drying methods on nutritional composition, physicochemical and functional properties of sweet potato leaves. J. Food Process Preserv. 43, e13884.

Sun, H.N., Mu, T.H. and Xi, L.S. (2016). Effect of pH, heat, and light treatments on the antioxidant activity of sweet potato leaf polyphenols. Int. J. Food Prop. 20, 318-332.

Sun, H.N., Mu, T.H., Xi, L.S., Zhang, M. and Chen, J.W. (2014). Sweet potato (Ipomoea batatas L.) leaves as nutritional and functional foods. Food Chem. 156, 380-389.

Toko, K. (2000). Taste sensor. Sensors Actuat. B: Chem. 64, 205-215.

Treftz, C., Zhang, F. and Omaye, S.T. (2015). Comparison between hydroponic and soil-grown strawberries: sensory attributes and correlations with nutrient content. Food Nutr. Sci. 06, 1371-1380.

Wang, A., Li, R., Ren, L., Gao, X., Zhang, Y., Ma, Z., Ma, D. and Luo, Y. (2018). A comparative metabolomics study of flavonoids in sweet potato with different flesh colors (Ipomoea batatas (L.) Lam). Food Chem. 260, 124134.

Wu, L., Gao, X., Xia, F., Joshi, J., Borza, T. and Wang-Pruski, G. (2019). Biostimulant and fungicidal effects of phosphite assessed by GC-TOF-MS analysis of potato leaf metabolome. Physiol. Mol. Plant Pathol. 106, 49-56.

Yang, C., Hu, Z., Lu, M., Li, P., Tan, J., Chen, M., Lv, H., Zhu, Y., Zhang, Y., Guo, L., Peng, Q., Dai, W. and Lin, Z. (2018). Application of metabolomics profiling in the analysis of metabolites and taste quality in different subtypes of white tea. Food Res. Int. 106, 909-919.

Zou, S., Wu, J., Shahid, M.Q., He, Y., Lin, S., Liu, Z. and Yang, X. (2020). Identification of key taste components in loquat using widely targeted metabolomics. Food Chem. 323,126822.

Zhu, G., Wang, S., Huang, Z., Zhang, S., Liao, Q., Zhang, C., Lin, T., Qin, M., Peng, M., Yang, C., Cao, X., Han, X., Wang, X., Van der Knaap, E., Zhang, Z., Cui, X., Klee, H., Fernie, A.R., Luo, J., and Huang, S. (2018). Rewiring of the fruit metabolome in tomato breeding. Cell. 172, 249-261. 


\section{Supplementary Data}

Table S1 Taste values of sweet potato leaves in eight cultivars under different growing patterns.

\begin{tabular}{lcccccccc}
\hline Cultivars & Fucaishu18 & Fushu7-6 & Tainong71 & Baisheng & Fushu24 & Guangshu87 & Jinshan57 & Fushu604 \\
\hline Soil cultures (S) & 6.00 & $5.50 \pm 1.08$ & $5.00 \pm 0.94$ & $1.00 \pm 0.67$ & $1.50 \pm 0.85$ & $1.00 \pm 0.67$ & $1.50 \pm 0.97$ & $4.50 \pm 1.27$ \\
\hline Hydroponics (H) & $8.00 \pm 0.82$ & $7.50 \pm 0.71$ & $8.00 \pm 1.25$ & $7.00 \pm 1.05$ & $4.50 \pm 1.90$ & $4.00 \pm 0.82$ & $5.00 \pm 1.41$ & $7.00 \pm 0.94$ \\
\hline Differences & 2.00 & 2.00 & 3.00 & 6.00 & 3.00 & 3.00 & 3.50 & 2.50 \\
\hline
\end{tabular}

Fucaishu18, Fushu7-6, Tainong71 are leafy sweetpotatoes for leaf vegetable, while other cultivars are common sweetpotatoes for tuber production.

The leaves of Fuchaishu18 growth in soil were used as the standard with defining its evaluation score as 6. 


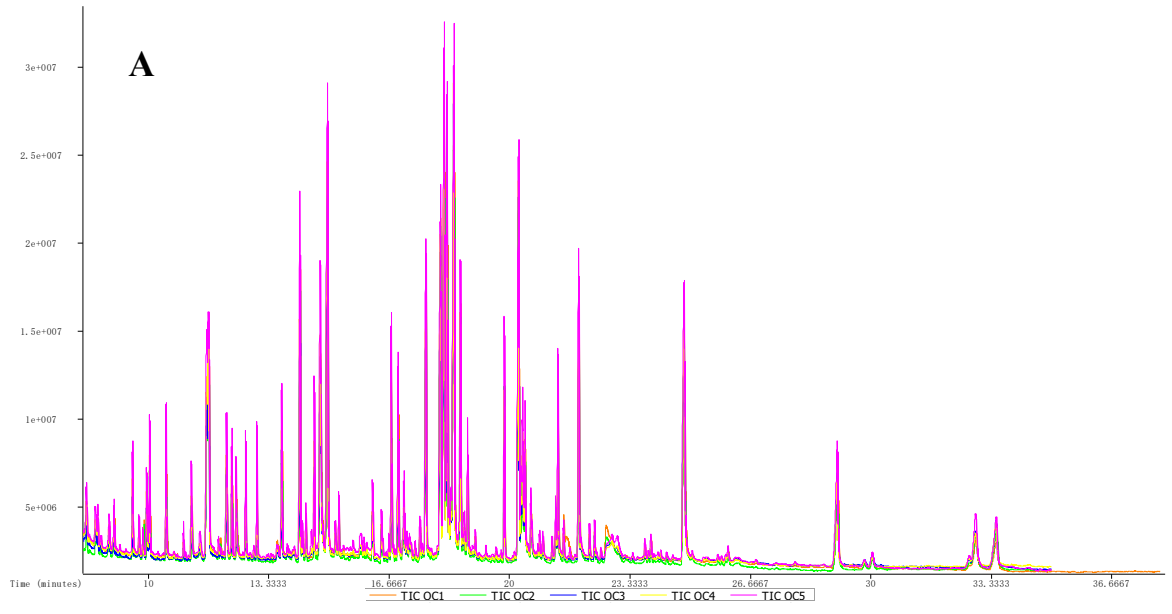

B

C

Fig. S1. Total Ions Chromatograph (TIC) of 5 Quality Control Samples (A), 5

Internal Standards in QC samples (B), and Blank sample (C). 


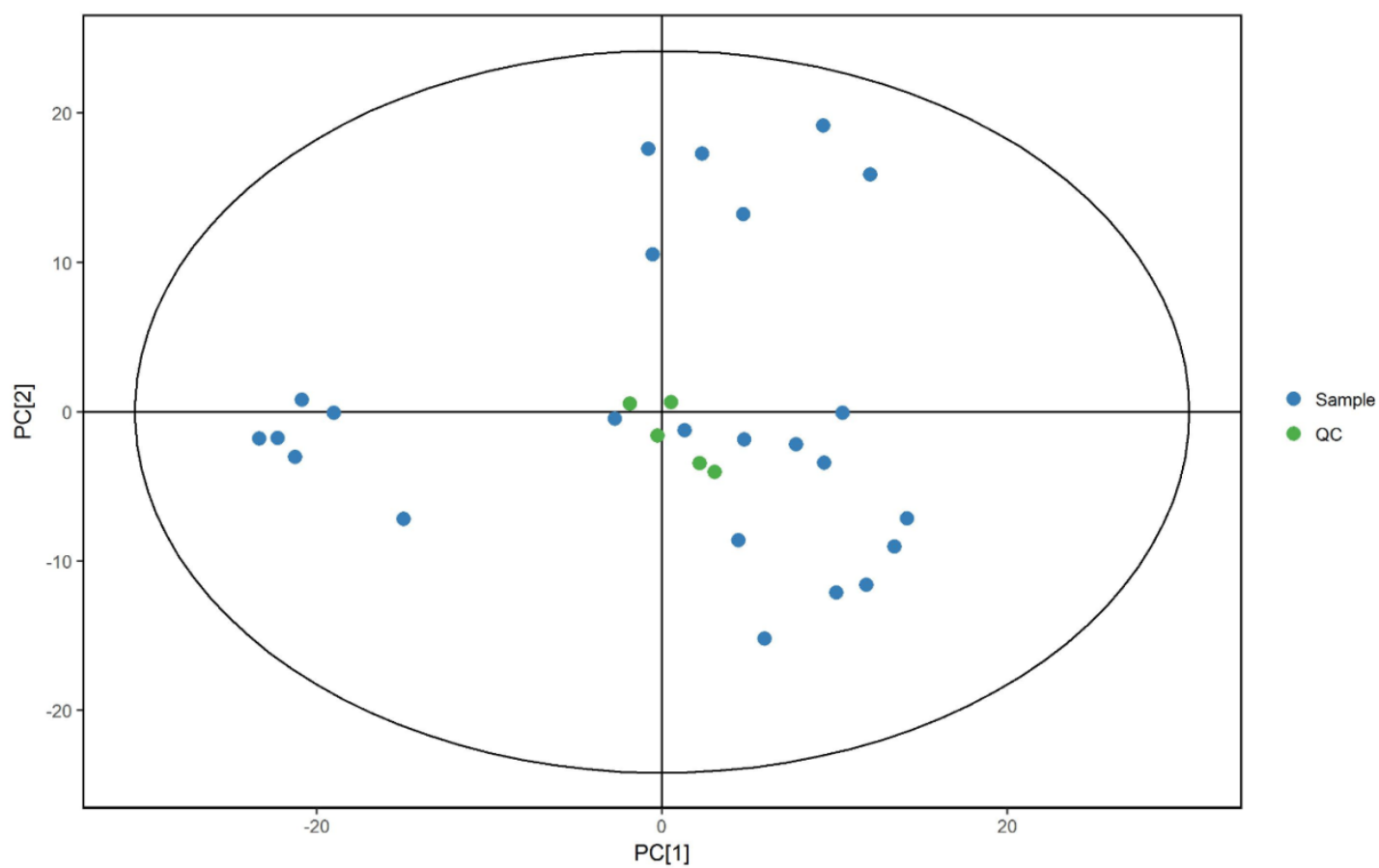

Fig. S2. Principal Component Analysis plot of the peaks in 5 QC samples and 24 test samples.

Green spots indicate the QC samples, while blue spots indicate the test samples. 


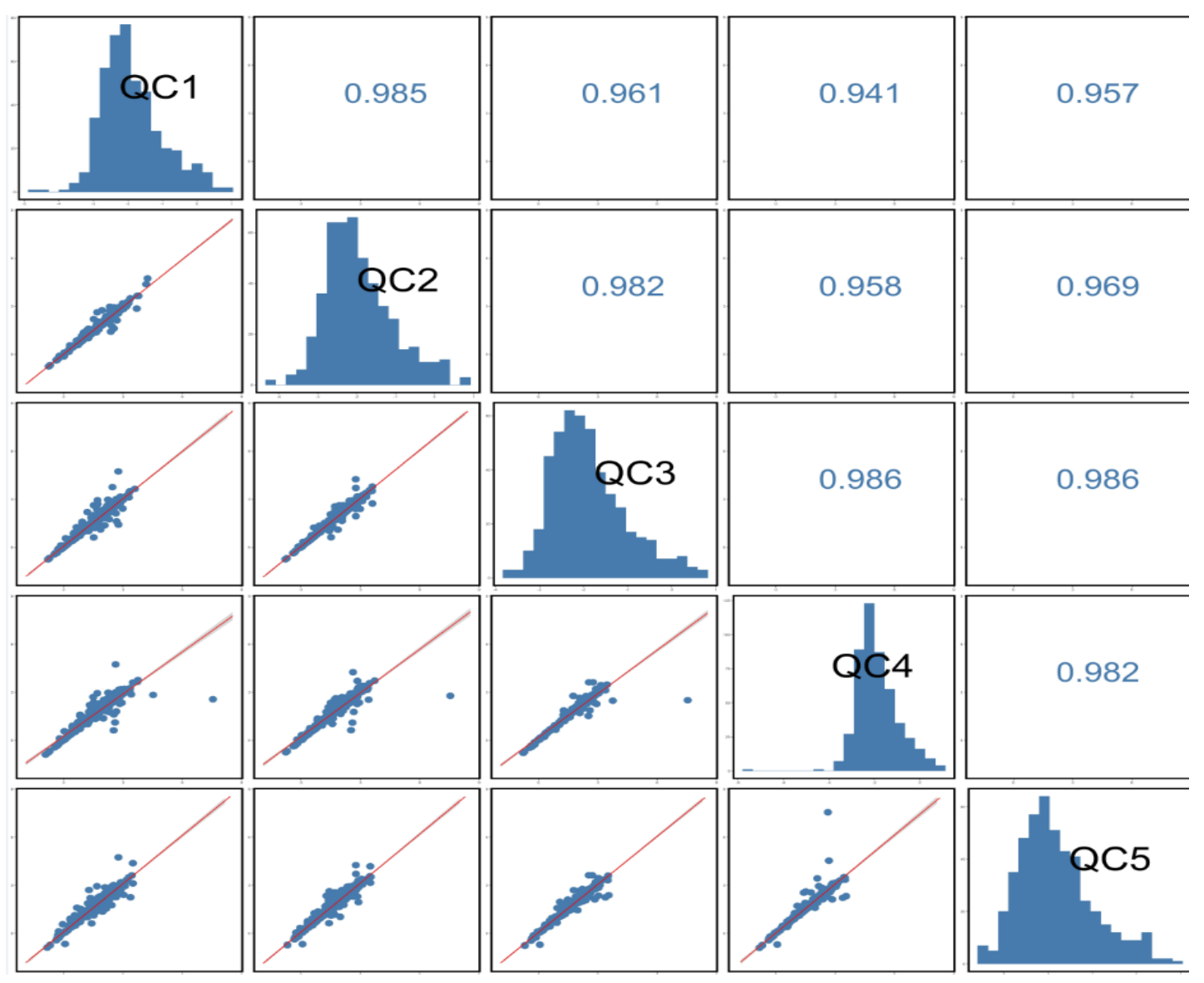

Fig. S3. High concordance among the peaks in five QC samples $\left(\mathrm{r}^{2} \geq 0.941\right)$. 

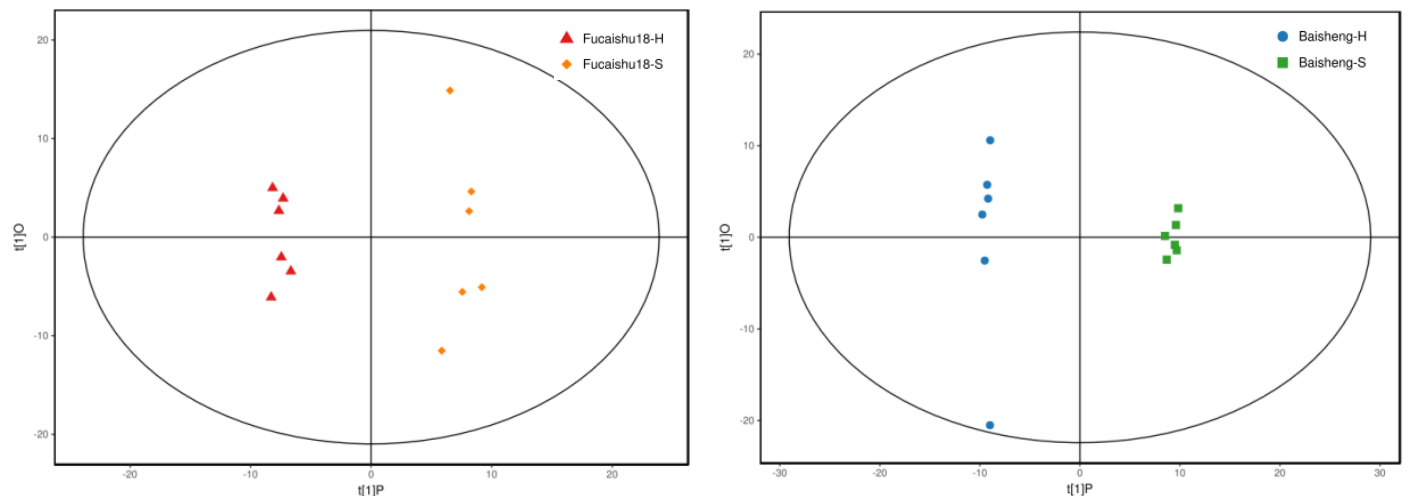

Fig. S4. Score scatter plot of OPLS-DA model for metabolites (peaks) in leaves of Fucaishu18(A) and Baisheng(B) under different growing patterns 

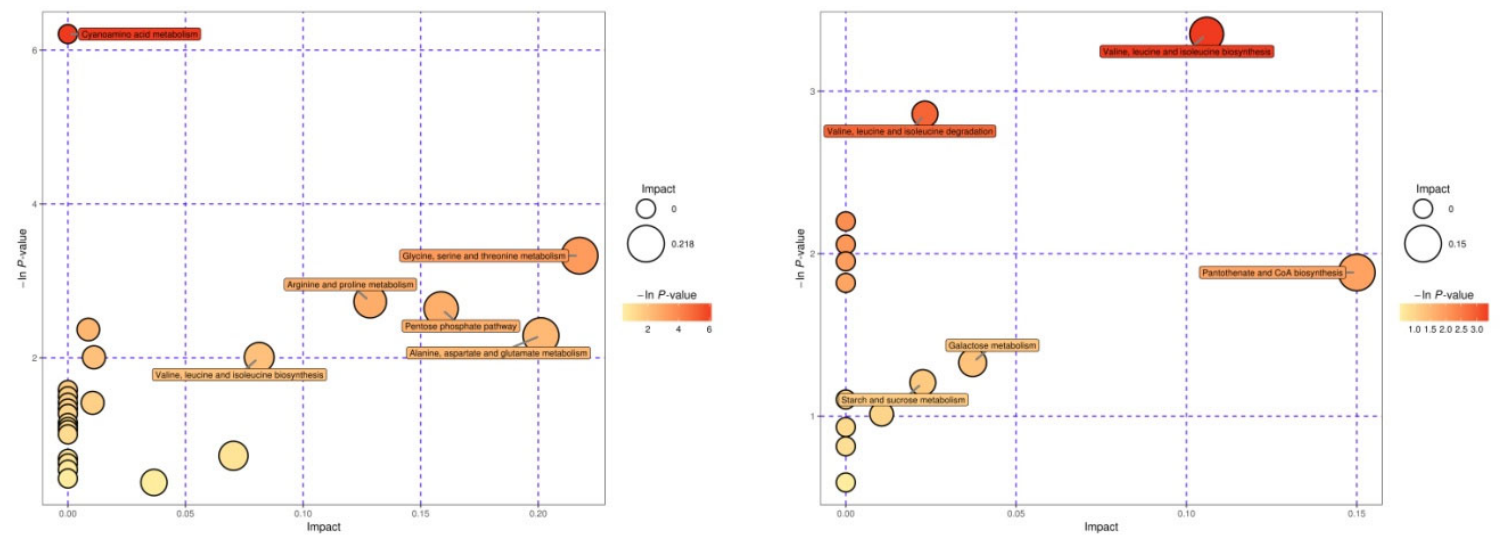

Fig. S5. Pathway analysis for different metabolites in leaves of Baisheng (A) and Fucaishu18 (B) under different growing patterns. 